\title{
Euphorbio meuselii-Rubetum ulmifolii, nuevo nombre para una asociación de zarzales de Sicilia (Italia)
}

\section{José Alfredo Vicente Orellana', Antonio Galán de Mera' \& Lorenzo Gianguzzi²}

${ }^{1}$ Laboratorio de Botánica, Facultad de Farmacia, Universidad CEU-San Pablo, apartado 67, 28660- Boadilla del Monte, Madrid, España. ${ }^{2}$ Department of Agricultural and Forest Sciences, University of Palermo, Via Archirafi 38, I-90123 Palermo, Italy.

\section{Correspondencia}

J. A. Vicente Orellana e-mail: avicore@ceu.es

Recibido: 18 julio 2018

Aceptado: 18 septiembre 2018

Publicado on-line: septiembre 2018
Euphorbio meuselii-Rubetum ulmifolii, a new name for a Sicilian association of brambles

Palabras clave: Nomenclatura, sintaxonomía, zarzales, Sicilia, Península Ibérica.

Key words: Nomenclature, Syntaxonomy, brambles, Sicily, Iberian Peninsula.
Durante el estudio de las comunidades de zarzales del occidente de la Península Ibérica y la Macaronesia (Vicente Orellana et al., 2016), hemos revisado los nombres de las asociaciones europeas (Weber, 1998), mediterráneas (Biondi et al., 2014) e ibéricas (Rivas-Martínez et al., 2001; Rivas-Martínez, 2011; Costa et al., 2012).

En un reciente trabajo sobre la vegetación forestal delnoroestedeSicilia, Gianguzzi etal. (2016) describieron la asociación Roso corymbiferaeRubetum ulmifolii (fig. 1; características: Crataegus monogyna Jacq., Euphorbia meuselii Geltman, Paeonia mascula subsp. russoi (Biv.) Cullen \& Heywood y Rubus ulmifolius Schott), mientras que más de 30 años antes Rivas-Martínez \& Arnaiz (Arnaiz, 1979) designaron con el nombre de Rubo ulmifolii-Rosetum corymbiferae a las rosaledas del interior de la Península lbérica (fig. 2; características y diferenciales: Rosa $x$ andegavensis Bastard, $R$. cariotii Chabert, $R$. corymbifera Borkh., $R$. micrantha Borrer ex Sm., $R$. pouziniiTratt. y Fraxinus angustifolia Vahl). Mientras que la asociación de Sicilia se comporta como una orla de los bosques de la asociación mesomediterránea superior subhúmeda superior Ampelodesmo mauritaniciQuercetum ilicis Gianguzzi, Cuttonaro, Cusimano \& Romano (Gianguzzi et al., 2016), la ibérica fue descrita como orla de la vegetación edafohigrófila, supra-mesomediterránea subhúmeda superior, de la asociación Fraxino angustifoliae-Quercetum pyrenaicae Rivas Goday 1964 corr. Rivas-Martínez, Fernández-González \& A. Molina 1988 (RivasMartínez et al., 1990), aunque también es etapa de sustitución de los bosques de la subalianza

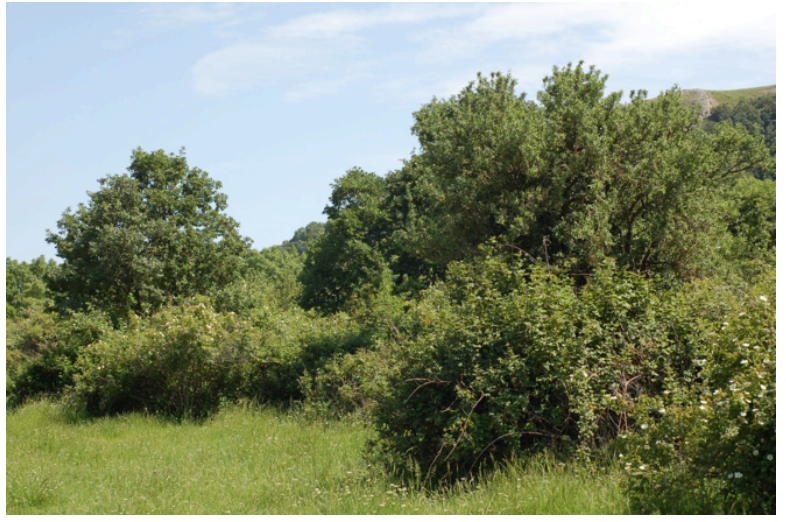

Figura 1. Euphorbio meuselii-Rubetum ulmifolii formando la orla de los bosques caducifolios en zonas montanas del occidente de Sicilia.

Figure 1. Euphorbio meuselii-Rubetum ulmifolii forming the boundary of deciduous forests in slopes and mountain zones of western Sicily.

Quercenion pyrenaicae Rivas-Martínez 1975.

El nombre Roso corymbiferae-Rubetum ulmifolii es inválido al interaccionar con Rubo ulmifolii-Rosetum corymbiferae, pues aunque sus nomenclaturas son inversas incumplen el artículo 32d del Código (Izco \& Del Arco, 2003), y por tanto se hace necesario elegir un nombre nuevo dentro del esquema fitosociológico de los zarzales de la isla de Sicilia: Euphorbio meuselii-Rubetum ulmifolii Vicente Orellana, Galán de Mera \& Gianguzzi nom. nov.

Euphorbia meuselii Geltman in Komarovia 


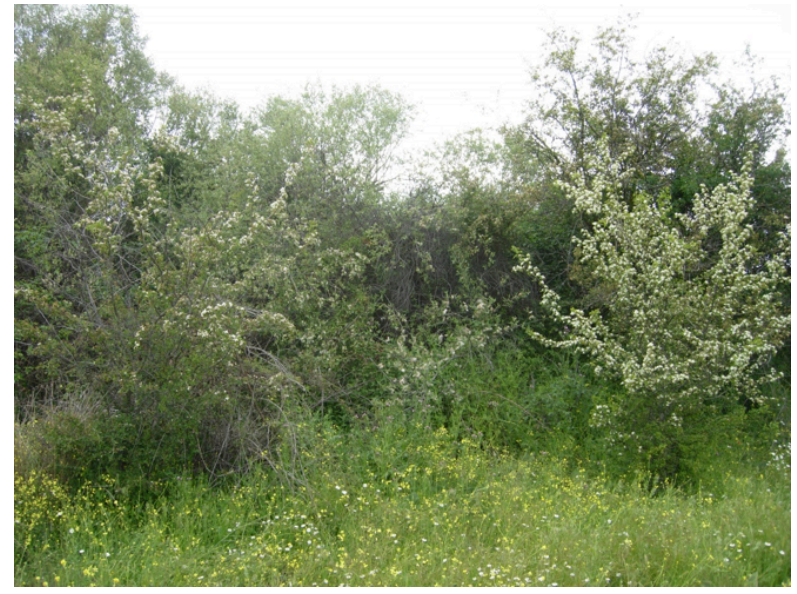

Figura 2. Rubo ulmifolii-Rosetum corymbiferae formando orla de los bosques esclerófilos y caducifolios en el centro de la Península Ibérica.

Figure 2. Rubo ulmifolii-Rosetum corymbiferae forming the boundary of deciduous and sclerophyllous forests in the centre of the Iberian Peninsula.

2: 24 (2002), [=Euphorbia meuselii Mazzola \& Raimondo in Bocconea 20: 11 (2007), nombre superfluo; Euphorbia amygdaloides subsp. arbuscula Meusel in Wiss. Z. Martin-Luther-Univ. Halle-Wittenberg, Math.-Naturwiss. Reihe 18: 134. (1969)] es una especie endémica del sur de Italia, presente en Calabria (desde Sila al Aspromonte) y en Sicilia, vicariante de E. amygdaloides L., de amplia distribución Euroasiática, distinguible la primera por sus tallos leñosos hacia la base, y sus hojas perennes y coriáceas (Pignatti, 1982). En Sicilia Euphorbia meuselii es típica de bosques mesófilos [Quercetea ilicis Br.-BI. ex A. \& O. Bolòs 1950, Querco-Fagetea Br.-Bl. \& Vlieger in Vlieger 1937, Salici-Populetea nigrae (Rivas-Martínez \& Cantó ex Rivas-Martínez, Báscones, T.E. Díaz, Fernández-González \& Loidi 1991) Rivas-Martínez \& Cantó 2002] y zarzales o espinares (RhamnoPrunetea Rivas Goday \& Borja ex Tüxen 1962) de colinas y zonas montañosas (Gianguzzi et al., 2016).

\section{RHAMNO-PRUNETEA Rivas Goday \& Borja ex Tüxen 1962 \\ + Prunetalia spinosae Tüxen 1952 \\ * Pruno-Rubion ulmifolii O. Bolòs 1954}

1. Euphorbio meuselii-Rubetum ulmifolii Vicente Orellana, Galán de Mera \& Gianguzzi nom. nov. [Roso corymbiferae-Rubetum ulmifolii Gianguzzi, Cuttonaro, Cusimano \& Romano in Plant Sociology
53(1): 30 (2016), tab. 17, inv. 3, non Rubo ulmifoliiRosetum corymbiferae Rivas-Martínez \& Arnaiz in Arnaiz, in Lazaroa 1: 131(1979)]

\section{Bibliografía}

Arnaiz, C. (1979). Ecología y fitosociología de los zarzales y espinales madrileños comprendidos en los sectores Guadarrámico, Manchego y Celtibérico-Alcarreño. Lazaroa, 1, 129-138.

Biondi, E., Blasi, C., Allegrezza, M., Anzellotti, I., Azzella, M.M., Carli, E., Casavecchia, S, Copiz, R., Del Vico, E., Facioni, L., Galdenzi, D., Gasparri, R., Lasen, C., Pesaresi, S., Poldini, L., Sburlino, G., Taffetani, F., Vagge, I., Zitti, S. \& Zivkovic, L. (2014). Plant communities of Italy: The Vegetation Prodrome. Plant Biosystems, 148(4), 728-814. DOI: 10.1080/11263504.2014.948527

Costa, J.C., Neto, C., Aguiar, C., Capelo, J., Espírito Santo, M.D., Honrado, J., Pinto-Gomez, C., Monteiro-Henriques, T., Sequeira, M., Lousã, M. (2012). Vascular plant communities in Portugal (Continental, Azores and Madeira). Global Geobotany, 2, 1-180. DOI: 10.5616/ gg 120001

Gianguzzi, L., Cuttonaro, P. \& Cusimano, D. (2016). Phytosociological survey vegetation map of Sicily (Mediterranean region). Journal of Maps, 12(5), 845-851. DOI: 10.1080/17445647.2015.1094969

Gianguzzi, L., Cuttonaro, P., Cusimano, D. \& Romano, S. (2016). Contribution to the phytosociological characterization of the forest vegetation of the Sicani Mountains (inland of north-western Sicily). Plant Sociology, 53(1), 5-43. DOI: 10.7338/pls2016531/02 Izco, J. \& Del Arco, M. (2003): Código internacional de nomenclatura fitosociológica. La Laguna, TF: Universidad de la Laguna. Pignatti, S. (1982). Florad'Italia. Vol.2.Bologna:Edagricole.

Rivas-Martínez S. (2011). Mapa de series, geoseries y geopermaseries de vegetación de España. Memoria del mapa de vegetación potencial de España. Itinera Geobotanica, 18(1/2), 5-800.

Rivas-Martínez, S., Fernández-González, F., Loidi, J., Lousã, M. \& Penas, A. (2001). Syntaxonomical checklist of vascular plant communities of Spain and Portugal to association level. Itinera Geobotanica, 14, 5-341.

Rivas-Martínez, S., Fernández-González, F., SánchezMata, D. \& Pizarro, J.M. (1990). Vegetación de la Sierra de Guadarrama. Itinera Geobotanica, 4, 3-132.

Vicente Orellana, J.A., Fernández de Castro, C., Vicente Orellana, J.A. \& Galán de Mera, A. (2016). A phytosociological classification of bramble communities in Macaronesia (Azores, Madeira and Canary Islands). Phytocoenologia, 46(2), 185-200. DOI: 10.1127/phyto/2016/0093

Weber, H.E. (1998). Outline of the vegetation of scrubs and hedges in the temperate and boreal zone of Europe. Itinera Geobotanica, 11, 85-120. 